\title{
INTRADERMOTERAPIA EM MELASMA
}

\author{
INTRADERMAL THERAPY IN MELASMA \\ Daniela Lopez ${ }^{1}$ \\ Fabiano de Abreu Rodrigues ${ }^{2}$
}

RESUMO: A intradermoterapia é uma técnica injetável, desenvolvida pelo médico francês Michel Pistor em 1952. Inicialmente seu objetivo era o uso em tratamentos médicos como doenças vasculares e infecciosas, lesões esportivas e para a melhoria da circulação. O melasma é uma disfunção caracterizada por manchas escuras, na face, braços, pescoço e colo, com maior frequência nas mulheres do que nos homens. Não há uma origem definida, podendo estar relacionada ao uso de anticoncepcionais, gestação e exposição solar. Sendo assim o objetivo do presente estudo foi identificar a ação da intradermoterapia com ativos específicos no tratamento do melasma. Tratase de uma revisão de literatura no portal de periódicos eletrônicos da SciELO, PubMed e Science Direct. Todos os ativos estudados apresentarem efeitos benéficos para o melasma, o ácido tranexâmico obteve destaque.

Palavras- chaves: melasma. Manchas escuras. Intradermoterapia, Cosmetologia. Mesoterapia.

\section{INTRODUÇÃO}

\section{I.I Intradermoterapia}

Assim como a mesoterapia, é uma técnica injetável, desenvolvida pelo médico francês Michel Pistor em 1952. Iinicialmente seu objetivo era o uso em tratamentos médicos como doenças vasculares e infecciosas, lesões esportivas e para a melhoria da circulação (Mammucari, 202I).

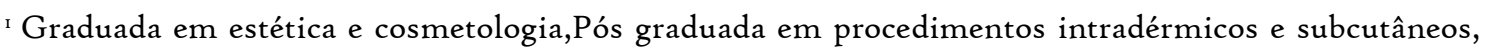
Especialista em Cosmiatria Orgânica e Ortomolecular, Presidente da sociedade brasileira de estética e cosmetologia, Pós graduada em biomedicina estética, Pós graduanda em Estética e Cosmetologia Avançada UNIFESP - Universidade Federal de São Paulo, Residência em Intradermoterapia Estética T.A.E Pesquisadora em cosmetologia em prol do Envelhecimento, curso integrado a UNICAMP. Email: daniela.lopezz@hotmail.com.

${ }^{2} \mathrm{PhD}$, neurocientista, mestre psicanalista, biólogo, historiador, antropólogo, com formações também em neuropsicologia, psicologia, neurolinguística, neuroplasticidade, inteligência artificial, neurociência aplicada à aprendizagem, filosofia, jornalismo e formação profissional em nutrição clínica - Diretor do Centro de Pesquisas e Análises Heráclito; Chefe do Departamento de Ciências e Tecnologia da Logos University International, UniLogos; Membro da Federação Européia de Neurociências e da Sociedade Brasileira e Portuguesa de Neurociências. Universidades em destaque: Logos University International, UniLogos, Nova de Lisboa, Faveni, edX Harvard, Universidad de Madrid.
} 
Atualmente é comumente utilizada na estética por meio de aplicações na derme ou hipoderme, através de agulha fina, devido a sua alta concentração de fármacos, atuando na má circulação, retenção de líquidos e inflamação dos tecidos conjuntivos dentre outros. As sessões duram em média de 30 a 60 minutos (Mammucari, 202I).

As substâncias aplicadas são em geral, extratos naturais, como: colágeno, ácido hialurônico, compostos à base de lecitina, vitaminas, minerais e coquetéis de aminoácidos no mesoderma, podendo conter até 50 ingredientes (Mammucari, 202I).

Após o recebimento da técnica o indivíduo não necessida de repouso ou afastamento de suas atividades laboratoriais. A técnica não é indicada em casos de alergias às substâncias utilizadas, doenças de pele no local de aplicação, cardíacos, gestantes e lactantes (Mammucari, 2020).

O tratamento é indolor, porém, podendo ocorrer vermelhidão nas regiões tratadas, passando espontaneamente após algumas horas. Reações alérgicas são raras (Mammucari, 2020).

\subsection{Melasma}

É uma disfunção caracterizada por manchas escuras na face, braços, pescoço e colo, com maior frequência nas mulheres do que nos homens. Não há uma origem definida, podendo estar relacionada ao uso de anticoncepcionais, gestação e exposição solar (Kwon, 2019).

A exposição solar, é acometida devido aos raios da luz ultravioleta que atingem a pele, bem como da luz visível (Kwon, 2019).

Os tratamentos para tal disfunção são variados, em todos os casos a orientação para a proteção contra raios ultravioleta e à luz visível. Cosméticos para uso tópico em casa, afim de melhorar o clareamento (Kwon, 2019).

Entre os tratamento mais utilizados são: peelings, intradermoterapia, fotoproteção, fotobiomodulação. Com o objetivo de clarear a pele, afim de impedir que o pigmento volte (Rajanala, 2019). 
O fotoprotetor, irá atuar na proteção contra os raios solares e luz visível. Indivíduos com melasma, precisam fazer uso diário de protetores solar, com ação contra os UVA e UVB (Rajanala, 2019).

Utilização de cosméticos para home care, à base de ácido glicólico, ácido retinóico, ácido azeláico, ácido kójico, ácido fítico, ácido tranexâmico e ácido dióico. Bem como a utilização da técnica de intradermoterapia para ingestão de ativos especifícos que irão atuar de uma maneira mais rápida. A utilização da fototerapia por meio dos laseres e luz intensa pulsada, com cuidado para não pior a pigmentação (Rajanala, 2019).

\section{Objetivo}

Identificar a ação da intradermoterapia com ativos específicos no tratamento do melasma.

\section{Metodologia}

Trata-se de uma revisão de literatura no portal de periódicos eletrônicos da SciELO, PubMed e Science Direct. Sendo utilizado os seguintes termos para realização da busca de dados em português: melasma, manchas escuras, intradermoterapia, cosmetologia e em inglês: melasma, dark spots, intradermotherapy, cosmetology.

4.Resultados e discussão

\begin{tabular}{|c|c|c|c|c|}
\hline Autor (ano) & Tipo de estudo & Amostra & Ativo & Análises \\
\hline $\begin{array}{c}\text { Karrabi } \\
(202 \mathrm{I})\end{array}$ & $\begin{array}{c}\text { Estudo clínico } \\
\text { randomizado } \\
\text { cego único }\end{array}$ & $\begin{array}{c}\text { 54 total } \\
\text { Grupor: intradermo com } \\
\text { ácido tranexâmico } \\
\text { Grupo2: creme de } \\
\text { cisteamina a 5\% }\end{array}$ & $\begin{array}{c}\text { ácido } \\
\text { tranexâmico }\end{array}$ & $\begin{array}{c}\text { Gravidade por } \\
\text { Dermacatch }{ }^{\circledR} \text { e o } \\
\text { Melasma Area } \\
\text { Severity Index } \\
\text { (mMASI) }\end{array}$ \\
\hline Iraji (2019) & Série de casos & 30 indivíduos & $\begin{array}{c}\text { 2\% ácido } \\
\text { tranexâmico } \\
\text { e 3\% ácido } \\
\text { ascórbico }\end{array}$ & $\begin{array}{c}\text { Área de Melasma } \\
\text { modificada e } \\
\text { Pontuação de } \\
\text { Gravidade } \\
\text { (mMASI) }\end{array}$ \\
\hline
\end{tabular}




\begin{tabular}{|c|c|c|c|c|}
\hline $\begin{array}{c}\text { Kaleem } \\
(2020)\end{array}$ & $\begin{array}{c}\text { Ensaio clínico } \\
\text { controlado }\end{array}$ & $\begin{array}{c}\text { 6o indivíduos } \\
\text { Gr: intradermo com ácido } \\
\text { tranexâmico } \\
\text { G2: Solução Salina } \\
\text { Normal (SN) }\end{array}$ & $\begin{array}{c}\text { Ácido } \\
\text { Tranexâmic } \\
\text { o }\end{array}$ & $\begin{array}{c}\text { Pontuação de } \\
\text { Gravidade } \\
\text { (mMASI) }\end{array}$ \\
\hline $\begin{array}{c}\text { Balevi } \\
(2017)\end{array}$ & $\begin{array}{c}\text { Ensaio clínico } \\
\text { controlado }\end{array}$ & $\begin{array}{c}\text { 5o indivíduos } \\
\text { com ácido salicílico e } \\
\text { vitamina c }\end{array}$ & $\begin{array}{c}\text { ácido } \\
\text { salicílico } \\
\text { combinado e } \\
\text { vitamina C }\end{array}$ & $\begin{array}{c}\text { qualidade de vida do } \\
\text { melasma } \\
\text { (MelasQoL) e } \\
\text { Pontuação de } \\
\text { Gravidade } \\
\text { (mMASI) }\end{array}$ \\
\hline
\end{tabular}

Fonte: Autores

No ensaio clínico de Karrabi et. al. 2021, ambos os grupos apresentaram resultados significativos nas análises de Gravidade por Dermacatch ${ }^{\circledR}$ e o Melasma Area Severity Index (mMASI), porém não signifativos entre os grupos.

Iraji et. al. 2019, avaliou os indivíduos após 12 semanas de tratamento, demonstrando resultados significativos na redução da pontuação mMASI em ambos os grupos.

Outro estudo da associação da intradermoterapia com o Ácido Tranexâmico, apresentou uma redução significativa na pontuação $H$-mMAS para o grupo com intradermoterapia. Após a técnica foi relatado eritema, inchaço e queimação em ambos os grupos, sendo considerados efeitos colaterais esperados (Kaleem, 2020).

Para os autores do ensaio clínico controlado de Balevi et. al. 2017, foi apresentado que os níveis de MelasQoL e MASI dos indivíduos em ambos os grupos diminuíram significativamente após o tratamento. E não houve nenhum efeito adverso em ambos os grupos.

\section{CONSIDERAÇÕES FINAIS}

Por meio dos ensaios clínicos incluídos nesta revisão de literatura, foi possível compreender os efeitos da técnica de intradermoterapia, o desenvolvimento da disfunção de melasma, bem como a relação de ambas, apensar de todos os ativos estudados apresentarem efeitos benéficos para o melasma. $O$ ácido tranexâmico obteve destaque. 


\section{REFERÊNCIAS}

MAMMUCARI, M., Maggiori, E., Antonaci, L., Fanelli, R., Giorgio, C., George, F., Mouhli, N., Intradermal therapy recommendations for standardization in localized pain management by the Italian Society of Mesotherapy. Minerva Med. n. II2, v. 2, págs.298-300, 202I doi: 10.23736/Soo26-4806.19.06278-5.

MAMmuCARI, M., Maggiori, E., Russo, D., Giorgio, C., Ronconi, G., Ferrara, P.E., Canzona, F., Antonaci, L., Violo, B., Mesotherapy: From Historical Notes to Scientific Evidence and Future Prospects. ScientificWorldJournal. n. io v. 20, e3542848, 2020 doi: $10.1155 / 2020 / 3542848$.

KWON, S.H., Na, J.I., Choi, J.Y., Park, K.C. Melasma: Updates and perspectives. Exp Dermatol. n. 28, v. 6, pags. 704-708, 2019 doi: 10.1111/exd.13844.

RAJANALA, S., Maymone, M.B.C., Vashi, N.A. Melasma pathogenesis: a review of the latest research, pathological findings, and investigational therapies. Dermatol Online J. n.15, v. Io, e13030, 2019

KARRABI, M., Mansournia, M.A., Sharestanaki, E., Abdollahnejad, Y., Sahebkar, M. Clinical evaluation of efficacy and tolerability of cysteamine $5 \%$ cream in comparison with tranexamic acid mesotherapy in subjects with melasma: a singleblind, randomized clinical trial study. Arch Dermatol Res. n. 313, v. 7, págs. 539-547, 202I doi: $10.1007 /$ s00403-020-02133-7.

IRAJI, F., Nasimi, M., Asilian, A., Faghihi, G., Mozafarpoor, S., Hafezi, H. Efficacy of mesotherapy with tranexamic acid and ascorbic acid with and without glutathione in treatment of melasma: A split face comparative trial. J Cosmet Dermatol. n. 8, 2019 doi: 1o.111//jocd.12874.

KALEEM, S., Ghafoor, R., Khan, S. Comparison of efficacy of Tranexamic Acid Mesotherapy versus $0.9 \%$ normal Saline for Melasma; A split face study in a Tertiary Care Hospital of Karachi. Pak J Med Sci. n. 36, v. 5, págs. 930-934, 2020 doi: I0.12669/pjms.36.5.2379.

BALEVI, A., Ustuner, P., Özdemir, M. Salicylic acid peeling combined with vitamin $C$ mesotherapy versus salicylic acid peeling alone in the treatment of mixed type melasma: A comparative study. J Cosmet Laser Ther. 2017 n.19, v. 5, págs. 294299. doi: 10.1080/14764172.2017.1314501. 